\title{
Biological role of postoperative low level laser therapy in preventing hydroxyapatite orbital implantation exposure: A case report
}

\author{
QI-HUA XU, YUE LI, JIN-HAI YU and HONG-FEI LIAO
}

The Affiliated Eye Hospital of Nanchang University, Nanchang, Jiangxi 330046, P.R. China

Received December 19, 2017; Accepted November 25, 2019

DOI: $10.3892 /$ etm.2021.9745

\begin{abstract}
Conjunctival sac stenosis is the contraction of the conjunctival sac as a result of trauma or disease. The aim of the present study was to observe the clinical effects of low-level laser therapy (LLLT) combined with hydroxyapatite (HA) orbital implantation as a treatment strategy for conjunctival sac stenosis. A total of 10 patients with conjunctival sac stenosis were treated with scleral graft transplantation in conjunction with HA implantation and postoperative LLLT. In addition, a rabbit model was used to investigate the biological mechanism underlying the effects of LLLT with the aim of preventing and treating orbital implantation exposure. The right eyeball was removed, orbital implantation performed and LLLT applied to experimental groups. ${ }^{99 \mathrm{~m} T c-M e t h y l}$ diphosphonate scanning methods were performed at different timepoints to compare the average radioactivity count of the region of interest between surgical (right) and control (left) eyes $(\mathrm{R} / \mathrm{L})$. Histopathological examination was performed 8 weeks post-surgery, followed by analysis of fiber vascularization. Following LLLT, moderate conjunctival wounds were completely healed within 2 weeks and severe stenosis wounds healed within 3 weeks. Following prosthesis implantation in the rabbit model, a significantly elevated $\mathrm{R} / \mathrm{L}$ ratio was observed after 4 weeks, whereas no significant difference was observed compared with the control group at 6 and 8 weeks postoperatively. Histopathological examination revealed that all implants were fibrotic. Overall, the present study demonstrated that LLLT promoted the survival of conjunctival grafts, stimulated conjunctival incision healing and promoted early vascularization of HA implants. Clinical trial registration no: ChiCTR-DDT-12002660 (www.chictr.org/cn/).
\end{abstract}

Correspondence to: Dr Hong-Fei Liao, The Affiliated Eye Hospital of Nanchang University, 463 Bayi Avenue, Nanchang, Jiangxi 330046, P.R. China

E-mail: 13970098689@126.com

Key words: postoperative complications, low-level laser therapy, vascularization, biological effect mechanism

\section{Introduction}

Conjunctival sac stenosis is the contraction of the conjunctival sac as a result of trauma or disease. Conjunctival sac stenosis is divided into congenital and posteriority, congenital micro-ophthalmia or nonocular disease, which may be present in combination with other deformities. The postnatal nature is largely due to chemical burns, conjunctival tissue defects caused by explosion injuries and the conjunctival sac stenosis caused by subsequent scar healing. Conjunctival sac narrowing is further classified into mild, moderate and severe: Mild conjunctival sac stenosis is defined as sac narrowing to less than one-third of the normal size and the sac and conjunctival dome becomes shallow; moderate conjunctival sac stenosis is defined as conjunctival sac narrowing to $\sim$ one-half the usual size; and severe conjunctival sac stenosis is defined as a conjunctival sac narrowing by more than one-third of the normal sac size, disappearance of the fornix and closure of the conjunctival $\operatorname{sac}(1)$.

At present, the standard treatment for conjunctival sac stenosis primarily involves mucosal or scleral transplantation and total eye reconstruction surgery $(2,3)$. These procedures are often unsuccessful due to infection, delayed orbital implant vascularization, exposure of the prosthetic socket and other disruptions of graft healing. In the present study, survival of the implant, wound healing of the conjunctival sac and the vascularization of the prosthesis were assessed, which are associated with surgical outcomes of conjunctival sac stenosis and postoperative complications (4,5). Low-level laser therapy (LLLT) does not require subsequent physical therapy. A number of studies have demonstrated positive outcomes of LLLT, including the promotion of wound healing $(6,7)$, reduction of inflammation $(8,9)$ and increased blood flow to local blood vessels (10).

The aims of the present study were to observe the clinical effects of LLLT combined with hydroxyapatite (HA) implantation in the treatment of conjunctival sac stenosis. Experiments in a rabbit model were simultaneously performed to observe the effects of LLLT on the vascularization of the prosthesis in animals. Together, the results of the present study highlight a possible mechanism by which LLLT may prevent exposure of the prosthesis. 


\section{Case report}

Ethics. The protocols used in the present study were approved by The Affiliated Eye Hospital of Nanchang University Ethics Committee (Nanchang, China). All participating patients provided informed written consent prior to inclusion in the present study.

Observation of clinical efficacy of LLLT in human patients. Clinical trial registration no: ChiCTR-DDT-12002660 (www. chictr.org/cn/). Between 2016 and 2017, 10 cases of conjunctival sac stenosis were investigated in 7 males and 3 females, aged 10-67 years old (average age, 36.5 years). Patients who had diseases which may have influenced wound healing, including diabetes, uremia, chronic inflammatory disease (acute or chronic infectious disease), endocrine disease, autoimmune disease, blood disease or tumor diseases were excluded. Patients were divided into mild, moderate and severe groups based on the severity of conjunctival sac narrowing and all patients underwent a scleral graft procedure, HA implantation combined with the eye 18-20 mm diameter. Following bandage removal 3 days after surgery, LLLT was administered. Patients in the moderate stenosis group were treated using two courses of LLLT, whereas patients in the severe group were treated using three courses of LLLT and the time interval between all treatments was 2 days. Survival of the sclera graft and conjunctival healing was observed at various time points after the operation. Antibiotics and epidermal growth factors were administered during laser treatment and follow-up and weekly check-ins were conducted to monitor the conjunctival incision until it was completely healed.

Postoperative LLLT following eye removal and HA orbital implantation in a rabbit model. A total of 30 male and female New Zealand white rabbits, weighing 2-3 kg, were used in the present study. HA implantations were $12 \mathrm{~mm}$ in diameter, had a $500 \mu \mathrm{m}$ pore size and a $100 \%$ interoperability rate (Beijing YHJ Science And Trade Co., Ltd.). The rabbits were randomly divided into the laser irradiation group (groups $\mathrm{B}$ and $C ; n=20$ ) and control group (group A, n=10). Each animal underwent right eye removal and HA prosthesis implantation. Irradiation groups B and C were treated with LLLT 3 days postoperatively; one course of treatment was given to group $\mathrm{B}$ and two courses of treatment were given to group $\mathrm{C}$ and the time interval between treatments was 1 week.

Laser probe and irradiation procedure. A semiconductor laser (JAM2-II type; Jiangxi Teli Anaesthesia \& Respiration Equipment Co., Ltd.) was used to issue radiation at a wavelength of $\lambda=650 \mathrm{~nm}$ with an adjustable power of $10 \mathrm{~mW}(0-20 \mathrm{~mW})$. The spot diameter was $10 \mathrm{~mm}$, with the laser power density at $12.7 \mathrm{~mW} / \mathrm{cm}^{2}$. Irradiation was performed once daily for $5 \mathrm{~min}$ at a dose of $\sim 3.8 \mathrm{~J} / \mathrm{cm}^{2}$ over a period of 5 days.

${ }^{99 m}$ Tc-Methyl diphosphonate $\left({ }^{99 m}\right.$ Tc-MDP) bone imaging examination in the rabbit model. To quantitatively assess the degree of vascularization following HA prosthesis implantation, a radionuclide scan was performed using single photon emission computed tomography (SPECT) on all the experimental animals at 1, 2, 4, 6 and 8 weeks after surgery. SPECT pretreatment conditions for peak $140 \mathrm{kev}$, window width is $15 \%, 256 \times 256$ static matrix, the preset count to $5 \times 10^{5}$ and the orbital symmetry position region of interest (ROI) was selected. The average radioactivity ratio was presented as $\mathrm{R} / \mathrm{L}$ and the experiments were repeated five times to calculate the average radioactivity ratio. Intravenous ${ }^{99} \mathrm{~m} \mathrm{Tc}-\mathrm{MDP}$ imaging agent $4 \mathrm{mCi}$ was injected and after $3 \mathrm{~h}$ the rabbits were anesthetized by animal intramuscular injection with ketamine $(50 \mathrm{mg} / \mathrm{kg}$ ) and chlorpromazine (25 mg/kg) (11) and laid on the test bench.

Histopathological examination of HA orbital implantation. The animals were sacrificed during the 8th postoperative week, with the connective tissue around their eyes observed. The implant was fixed for $24 \mathrm{~h}$ with formaldehyde and after $72 \mathrm{~h}$ the nitrate was removed. Conventional paraffin embedding, sagittal section and H\&E staining were all performed respectively. The fibrovascular and inflammatory cell infiltration in the LLLT groups and control group were observed under an optical microscope (magnification, $\mathrm{x} 4$ and $\mathrm{x} 40$ ).

Statistical analysis. Statistical analyses were performed using SPSS software (IBM Corp.). The data are expressed as the mean \pm standard deviation (unless otherwise shown). The average radioactivity count ratio of ROI between surgical eyes and control eyes $(\mathrm{R} / \mathrm{L})$ in groups $\mathrm{A}, \mathrm{B}$ and $\mathrm{C}$ was compared at different time points using one-way ANOVA. After the comparison, t-test was used between the groups. $\mathrm{P}<0.05$ was considered to indicate a statistically significant difference.

\section{Results}

Patients treated with LLLT exhibit favorable postoperative conjunctival healing. Patient information, diagnosis and treatment and postoperative conjunctival healing data are presented in Table I and clinical manifestations of conjunctival sac stenosis are depicted in Figs. 1-3. All 10 patients received LLLT on four different occasions. Observation of conjunctival incision healing at different time points following laser treatment revealed new blood vessel formation, granulation tissue hyperplasia, reduced secretion and relieved conjunctival congestion edema. After 2 weeks post LLLT, it was observed that the conjunctival incision was fully healed in patients with moderate conjunctival sac stenosis, whereas complete healing of the incision in patients with severe conjunctival sac stenosis was observed at 3 weeks following LLLT. During the follow-up, the conjunctival wound healing of each group was assessed, and all incisions were observed to be clean with no secretions or complications.

LLLT in a rabbit model demonstrates favorable postoperative healing following HA implantation. All 30 animals survived the experiments. Following HA implantation, good conjunctival wound healing was observed with no secretions and the site was clean. No signs of infection, eye exposure or other complications were observed. After 8 weeks post LLLT, normal eye movement was observed.

${ }^{99 m} T c-M D P$ imaging in LLLT treated rabbits increases the radioactivity count ratio in $R O I$. The average radioactivity count ratio in the ROI was significantly increased in LLLT groups B and C compared with the control group A at 1,2 and 4 weeks post LLLT 


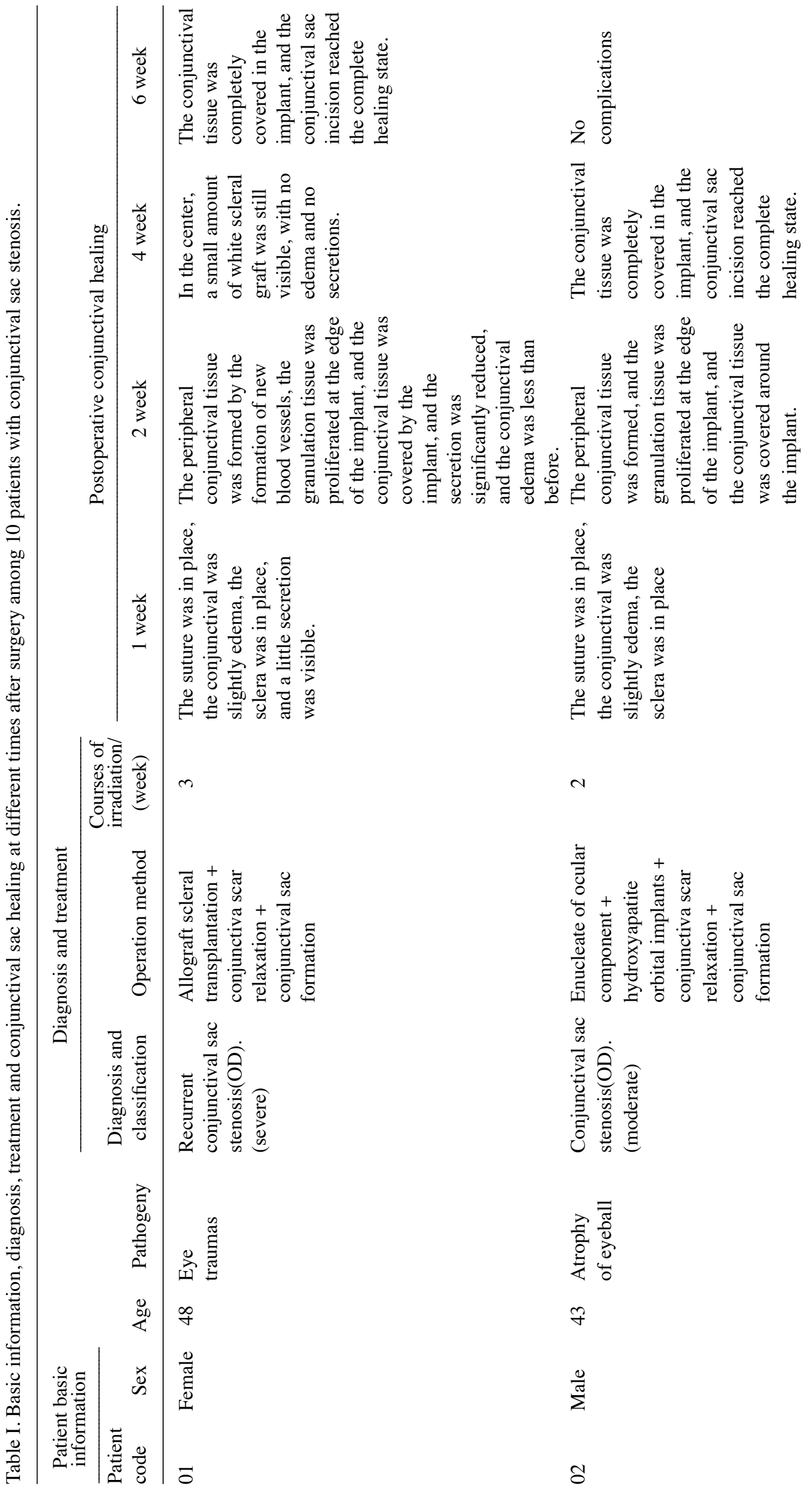




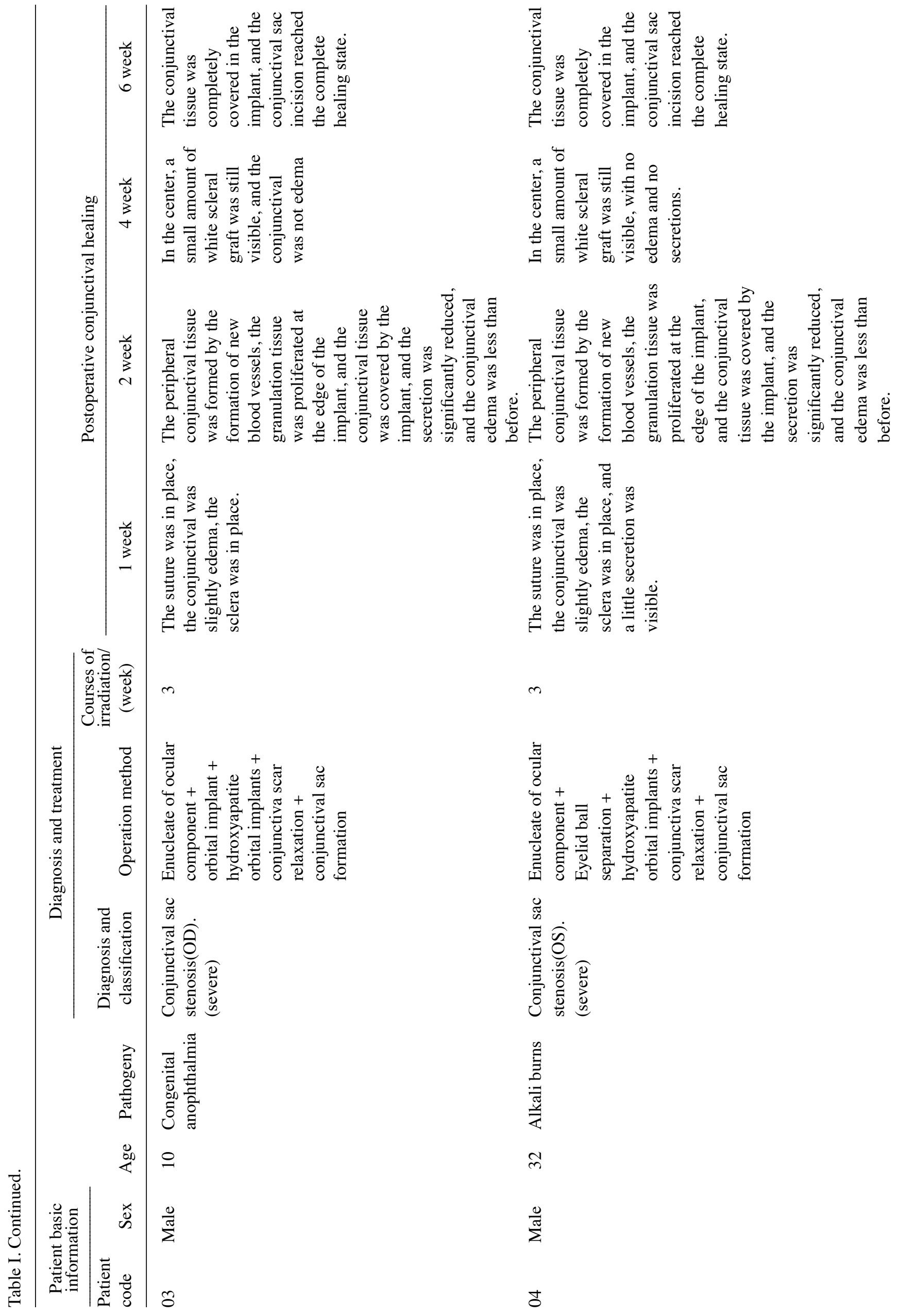




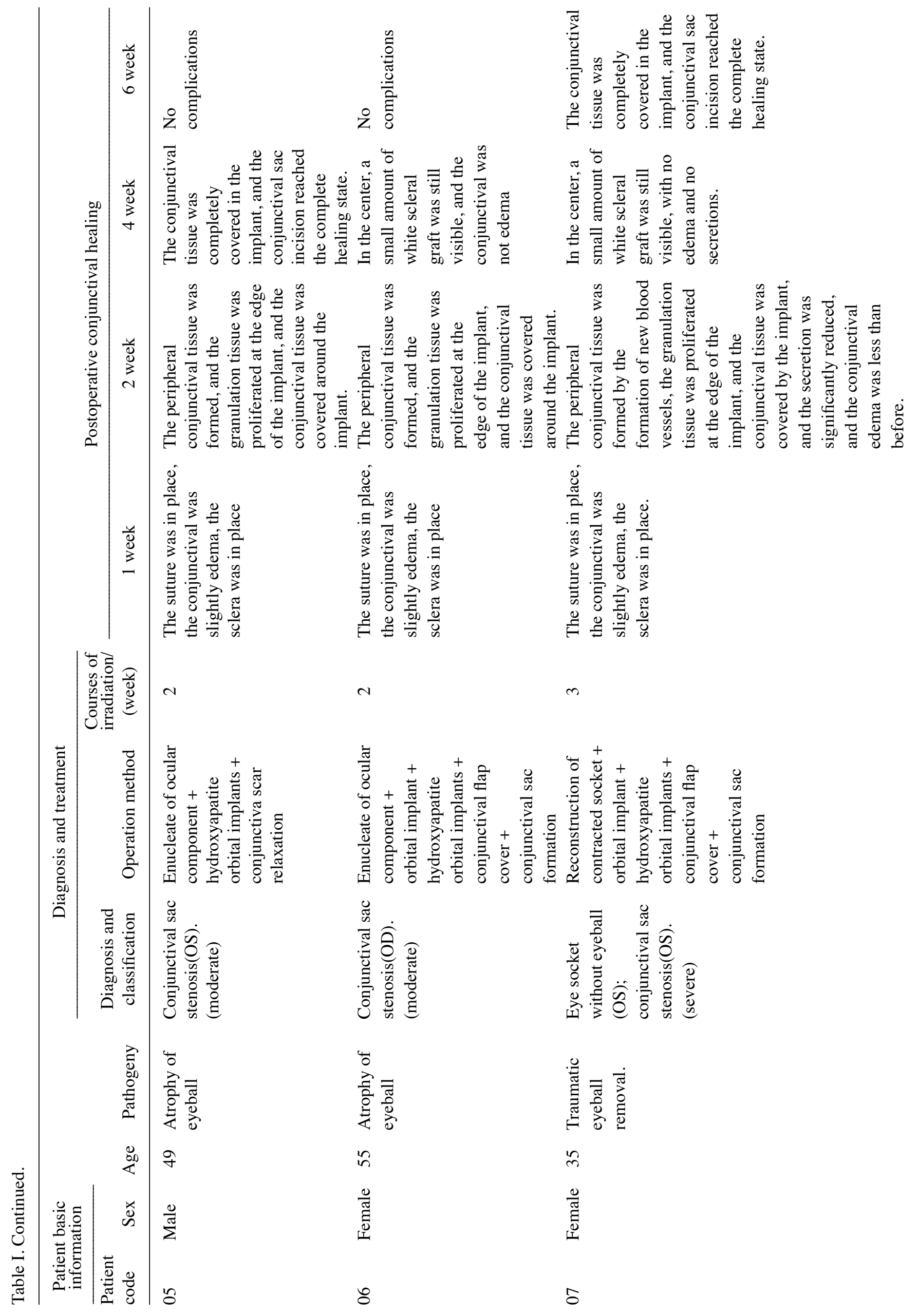




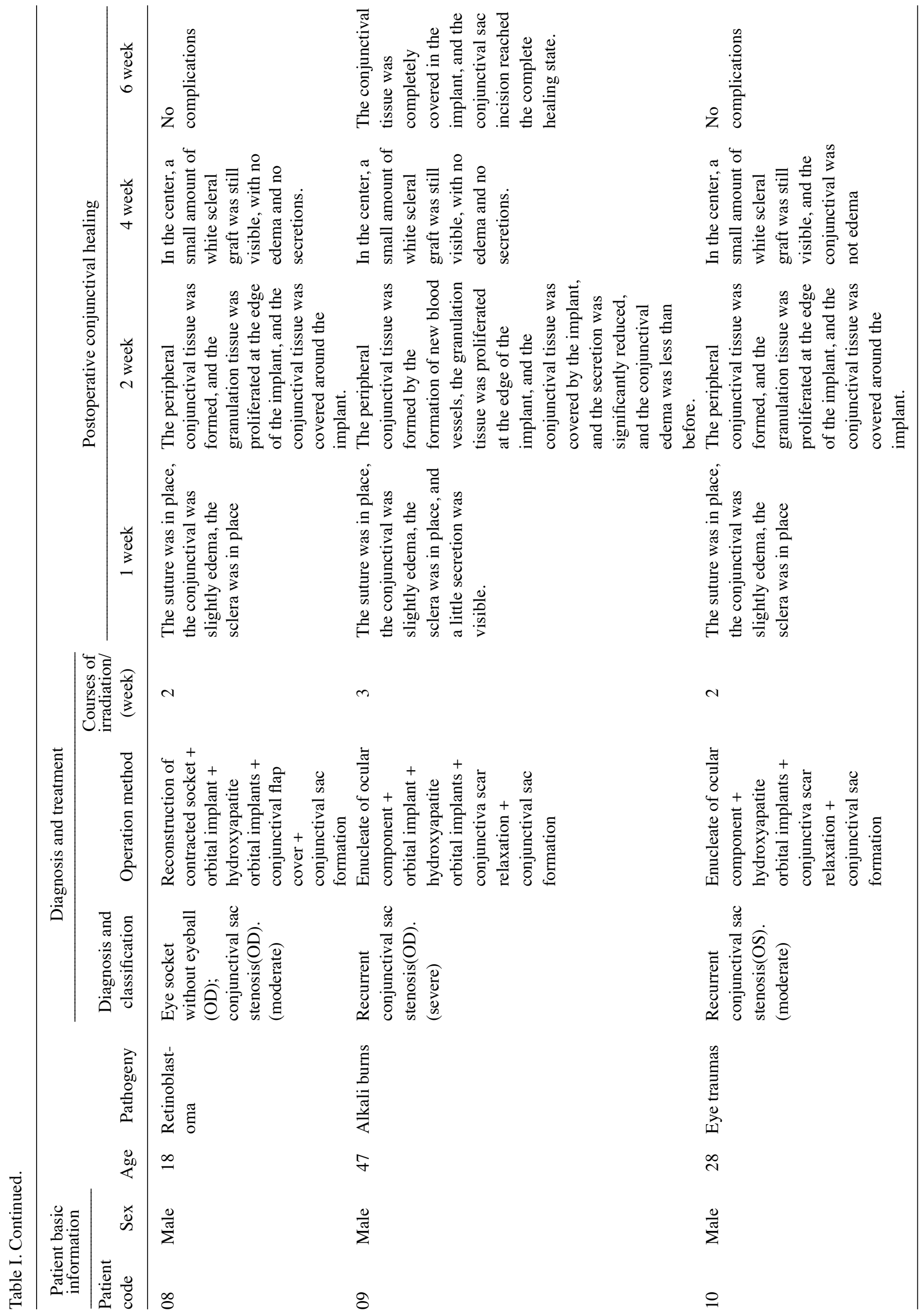




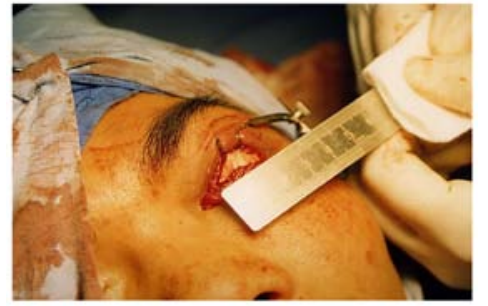

Figure 1. Intraoperative: The patient's left eye operated on using HA orbital implant and allograft patch repair. The eye socket is visibly full, peripheral conjunctival tissue exhibited congestion, edema and residual conjunctival edge was stitched on the peripheral part of the graft and the central wide range of dental exposed.

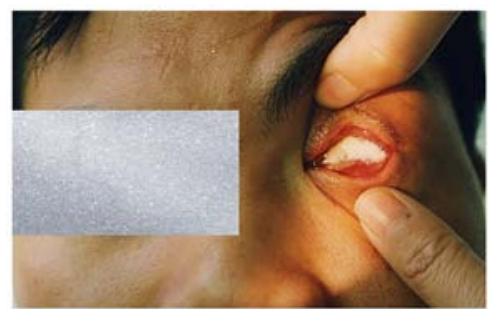

Figure 2. After 1 week of laser irradiation treatment, conjunctival hyperemia and edema had subsided and there were no obvious secretions. The conjunctiva was vasodilated, neovascular bud growth was observed and conjunctival tissue began to spread to the graft area.

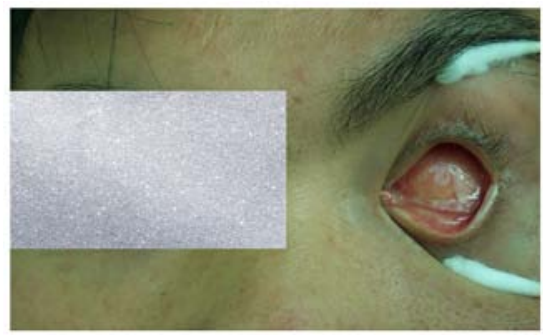

Figure 3. After 1 month of laser treatment, there were no obvious secretions of the eye, conjunctival hyperemia and edema had completely subsided and the conjunctival tissue completely covered the graft area, with a healing state achieved.

( $\mathrm{P}>0.05)$. There was no significant difference in the average radioactivity count ratio in the ROI between groups $\mathrm{B}$ and $\mathrm{C}$. In addition, there was no significant difference in the average radiological count $(\mathrm{R} / \mathrm{L})$ in groups $\mathrm{A}, \mathrm{B}$ and $\mathrm{C}$ at 6 and 8 weeks post-surgery $(\mathrm{P}<0.05$; Figs. 4 and 6$)$. The average $\mathrm{R} / \mathrm{L}$ values of LLLT groups $\mathrm{B}$ and $\mathrm{C}$ were higher compared with the control group A between 1 and 4 weeks post-surgery, whereas at 6 and 8 weeks, no significant difference was observed between the 3 groups.

Histopathological examination of HA implants in LLLT rabbits demonstrated generation of new fibrovascular tissue. In the 8th postoperative week, the HA implants were examined, and the implants were surrounded by fibrovascular tissues, sclera and inflammatory cells. Sagittal section H\&E staining displayed formation of new fibrovascular tissues in the HA implants and no obvious inflammatory cell infiltration in LLLT groups B and C. In the control group A, only new vascular fibrous tissues were observed around the orbital implants (Fig. 5).

\section{Discussion}

The treatment of conjunctival sac stenosis is difficult; a number of studies have reported the use of variants or autologous tissue slices for repair, such as autologous sclera, fascia, conjunctiva, hard palate mucosa, decellularized dermal, amniotic membrane, temporal shallow artery temporalis fascia island flap, but cases of postoperative restenosis in the conjunctival sac and implant exposure continue to be reported (2). Additional factors also contribute to the complexity of conjunctival sac stenosis, including a lack of conjunctival epithelial cells around conjunctival epithelial hyperplasia of the cell division that require repair, with several patients possessing conjunctival tissues with slow growth rates post injury. Often, the healing time and high conjunctival surface tension results in dissolving of the allograft or autograph phenomenon (3) and loss of conjunctival tissue carrier support can lead to complete failure due to postoperative infection.

In the event of extensive conjunctival destruction caused by conjunctival sac narrowing, treatment is largely limited to surgery with the severity of stenosis determining the procedure performed. The primary purpose of treatment is to restore the form of the patients conjunctival sac. Moderate and severe conjunctival sac narrowing is commonly caused by chemical injury, burns or other causes of conjunctival sac of scar contracture and is characterized by narrowing of the dome, which is often repaired by transplantation. Previous studies have investigated the use of an allograft or autograft as a means for conjunctival sac repair; however, as the transplant can only be used as a basement membrane, a lack of conjunctival epithelial cell division and proliferation can extend the conjunctival incision healing time (12). Meanwhile, in cases where the conjunctival surface tension is increased due to excessive allograft or autograft dissolution phenomena, exposure of orbital implants can cause procedure failure $(12,13)$.

In the present study, patients with moderate and severe conjunctival sac stenosis received a conjunctival sac scleral graft implantation and HA implants to fill the eye socket and to restore conjunctival sac form. HA is a commonly used filling material and its clinical application has permitted rapid development in orbital plastic surgery. The porous structure of HA is beneficial for the growth of vascular fibers and rapid vascularization is the basis of successful orbital implantation. In the present study, LLLT was performed post-surgery to promote the biological effects of wound healing and to observe its clinical therapeutic effect on conjunctival sac stenosis. The results showed that the local inflammatory symptoms after treatment were significantly reduced. The use of LLLT can stimulate conjunctival blood vessels around the wound, increase the speed of local fascia tissue formation and vascularization, promote conjunctival epithelial cell growth to cover the surface of the implant, promote faster wound healing and thus reduce postoperative follow-up complications.

In the present study, LLLT was employed for adjuvant treatment following application of the regular operation method and the mechanism underlying promotion of tissue healing and the accompanying curative effects have been previously reported (14). Hornig et al (15) demonstrated that LLLT promoted tissue healing by stimulating fibroblast activity, whereas de Araújo et al (16) investigated the effects of He-Ne laser irradiation on wound healing in mice demonstrating wound inflammation relief, accelerated fibroblast activation and an increased number of collagen fibers in irradiated 

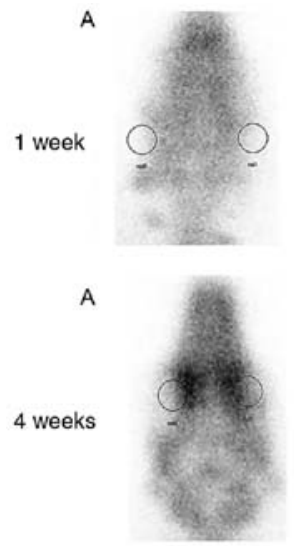

8 weeks

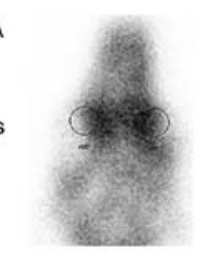

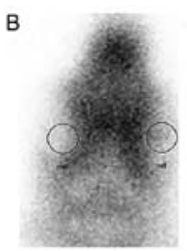

B

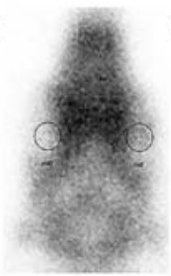

B

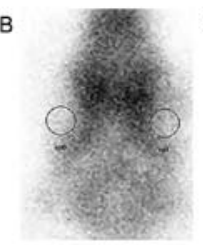

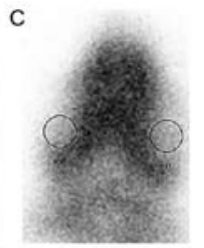

C

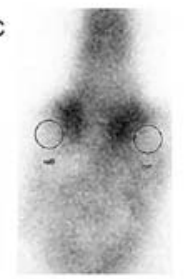

C

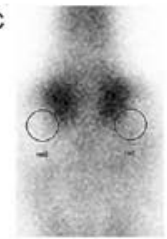

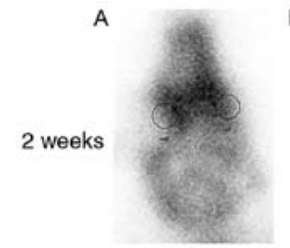
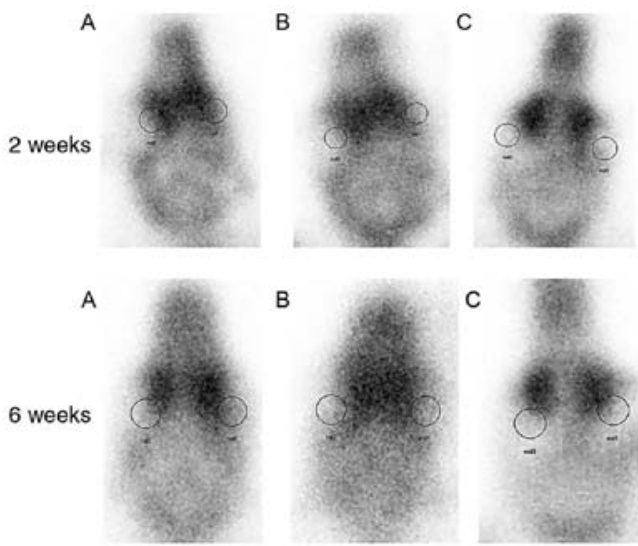

Figure 4. After 1 week post HA orbital implantation, the average R/L of group B and C was higher compared with group A. After 2 weeks post HA orbital implantation, the average $\mathrm{R} / \mathrm{L}$ of group $\mathrm{B}$ and $\mathrm{C}$ was higher compared with group $\mathrm{A}$. After 4 weeks post $\mathrm{HA}$ orbital implantation, the average R/L of group $\mathrm{B}$ and $\mathrm{C}$ was higher compared with group A. After 6 weeks post HA orbital implantation, there was no difference detected in average R/L between 3 groups. After 8 weeks post HA orbital implantation, there was no significant difference detected in regard to the average R/L between three groups. HA, hydroxyapatite; R/L, the average radioactivity count of the region of interest between surgical (right) and control (left) eyes; group A; control group underwent right eye removal and HA orbital implantation only $(n=10)$; group B, underwent right eye removal and HA orbital implantation followed by one course of LLLT 3 days post-surgery $(n=10)$; group $C$, underwent right eye removal and HA orbital implantation followed by two courses of LLLT with a time interval of 1 week between treatments starting 3 days post-surgery $(n=10)$; roi0, region of interest 0 ; roil, region of interest 1 .

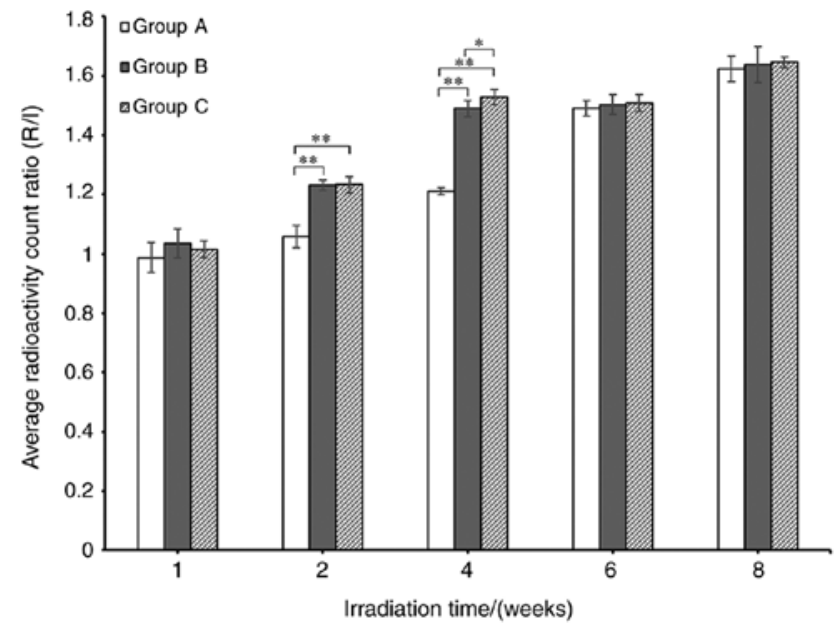

Figure 5. Average radioactivity count ratio R/L of region of interest at different time points post irradiation. Values are expressed as the mean \pm standard error of the mean. ${ }^{*} \mathrm{P}<0.05,{ }^{* *} \mathrm{P}<0.01$. Group A control group underwent right eye removal and HA orbital implantation only. Group B underwent right eye removal and HA orbital implantation followed by one course of LLLT 3 days post-surgery. Group $\mathrm{C}$ underwent right eye removal and HA orbital implantation followed by two courses of LLLT with a time interval of 1 week between treatments starting 3 days post-surgery. $n=10$ /group. $R / L$, the average radioactivity count of the region of interest between surgical (right) and control (left) eyes; LLLT, low-level laser therapy.

areas. A previous study utilized LLLT on traumatic tympanic membrane perforation and demonstrated that LLLT influenced wound healing by inducing changes in tympanic membrane proliferation activity, increasing squamous cell hyperplasia, cell migration and fibroblast proliferation as well as increasing

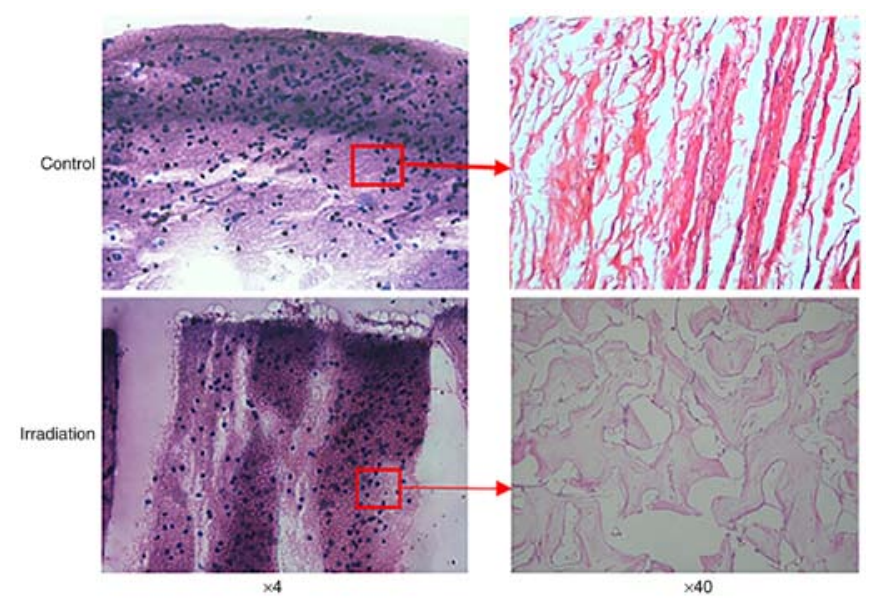

Figure 6. Histopathological examination 8 weeks post right eye removal and HA orbital implantation in the control and irradiation groups in the rabbit model. Animals in the irradiation groups received one or two courses of LLLT starting 3 days post-surgery with a time interval of 1 week between treatments if appropriate. In the irradiation groups, dense collagen fibers were observed in the center of the implantation indicative of fresh angiogenesis with no signs of inflammatory cell infiltration were observed, whereas, in the control group, only new vascular fibrous tissue was observed around the orbital implants. Magnification, $\mathrm{x} 4$ and x40.

vascularization and this study concluded that the use of LLLT increased matrix and collagen synthesis and promoted fibrous tissue regeneration, ultimately reducing the healing time post tympanic membrane perforations (17).

Based on previous studies and the results obtained in the present study, it was concluded that LLLT can be applied as a postoperative adjuvant therapy to promote healing, improve 
graft survival and conjunctival incision healing in patients with conjunctival sac stenosis. The mechanisms underlying these favorable postoperative outcomes may include: Promotion of conjunctival epithelial cell proliferation and migration, thus accelerating plant of conjunctival tissue to center around the crawling; promotion of local microcirculation; promotion of absorption of exudate in the human body and alleviation of inflammation, stimulating fibroblast proliferation and collagen formation beneficial for tissue repair and wound healing; and promotion of vascularization of the HA implant. In the present study, the influence of LLLT on the vascularization of the HA implant was widely observed. The results of animal experiments demonstrated that there were numerous new fibrous vascular tissues formed around the eye seat of implant, sclera shell and the eye muscle attachment and the surface of the sphere could be observed in the perforation of the fiber vessels. Radionuclide scanning also revealed that vascularization in the prosthetic eye following LLLT was faster compared with the control group. Overall, it was found that LLLT promotes the success of conjunctival grafts and conjunctival incision healing in patients with conjunctival sac stenosis. In addition, LLLT significantly promotes the early vascularization of HA implants. However, further investigation to resolve the underlying mechanism of LLLT promoting tissue healing, prevention and the control of ocular surface exposure postoperatively in patients with conjunctival sac stenosis is required to further validate the findings of the present study.

\section{Acknowledgements}

Not applicable.

\section{Funding}

This research was supported by the Jiangxi Provincial Key R\& D Program (20181BBG70007) and the Jiangxi Natural Science Foundation Project (20181BAB205035).

\section{Availability of data and materials}

The datasets used and/or analyzed during the present study are available from the corresponding author on reasonable request.

\section{Authors' contributions}

QX and YL performed experiments, data collation and analysis, and writing the manuscript and modification of the study. YJ and YL together completed the animal experimental part of the manuscript, including the experimental process of animal feeding, animal surgery and tissue section processing so YJ as the second author of the article. HL designed the study, performed the surgeries and revised the manuscript.

\section{Ethical approval and consent to participate}

The study protocol was approved by The Affiliated Eye Hospital of Nanchang University Ethics Committee (Nanchang, China). All participating patients provided informed written consent documentation prior to inclusion in the present study.

\section{Patient consent for publication}

Patients or patients' guardians provided consent for the publication of images in the present study.

\section{Competing interests}

The authors declare that they have no competing interests.

\section{References}

1. Hong Y, Xu GX and Chen SD: Hydroxyapatite orbital implantation combined with united skin grafting for eye-socked depression with $\mathrm{III}^{\circ}$ conjunctival sac stenosis, 2008.

2. Kolli S, Ahmad S, Lako M and Figueiredo F: Successful clinical implementation of corneal epithelial stem cell therapy for treatment of unilateral limbal stem cell deficiency. Stem Cells 28: 597-610,2010.

3. Li D, Jie Y, Liu H, Liu J, Zhu Z and Mao C: Reconstruction of anophthalmic orbits and contracted eye sockets with microvascular radial forearm free flaps. Ophthalmic Plast Reconstr Surg 24: 94-97, 2008.

4. Tuby H, Maltz L and Oron U: Implantation of low-level laser irradiated mesenchymal stem cells into the infarcted rat heart is associated with reduction in infarct size and enhanced angiogenesis. Photomed Laser Surg 27: 227-233, 2009.

5. Corazza AV,Jorge J,Kurachi Cand Bagnato VS: Photobiomodulation on the angiogenesis of skin wounds in rats using different light sources. Photomed Laser Surg 25: 102-106, 2007.

6. Pinheiro AL, Meireles GC, de Barros Vieira AL, Almeida D, Carvalho CM and dos Santos JN: Phototherapy improves healing of cutaneous wounds in nourished and undernourished Wistar rats. Braz Dent J 15: SI21-SI28, 2004.

7. Ankri R, Lubart R and Taitelbaum H: Estimation of the optimal wavelengths for laser-induced wound healing. Lasers Surg Med 42: 760-764, 2010.

8. Correa F, Lopes Martins RA, Correa JC, Iversen VV, Joenson J and Bjordal JM: Low-level laser therapy (GaAs lambda=904 nm) reduces inflammatory cell migration in mice with lipopolysaccharide-induced peritonitis. Photomed Laser Surg 25: 245-249, 2007.

9. Mesquita-Ferrari RA, Martins MD, Silva JA Jr, da Silva TD, Piovesan RF, Pavesi VC, Bussadori SK and Fernandes KP: Effects of low-level laser therapy on expression of TNF- $\alpha$ and TGF- $\beta$ in skeletal muscle during the repair process. Lasers Med Sci 26: 335-340, 2011

10. Bossini PS, Fangel R, Habenschus RM, Renno AC, Benze B, Zuanon JA, Neto CB and Parizotto NA: Low-level laser therapy $(670 \mathrm{~nm})$ on viability of random skin flap in rats. Lasers Med Sci 24: 209-213, 2009.

11. Chen Q: Establishing and studying the model of posterior capsule opacification in rabbits. Compilation of the 9th Central and Southern Region Experimental Animal Science and Technology Exchange Conference, pp1016-1021, 2009.

12. Gu J, Zhai J, Liao G and Chen J: Boston type I keratoprosthesis implantation following autologous submandibular gland transplantation for end stage ocular surface disorders. Ocul Immunol Inflamm 26: 452-455, 2018.

13. Liu DY: Clinical analysis of 129 cases of traumatic conjunctival sac stenosis. J Ocul Injury Occup Ophthalmol 32: 770-771, 2010.

14. Nie YH, Xing YQ, Guo Y and Zhu J: Analysis on the causes and management of exposure of orbital hydroxyapatite implants. Recent Adv Ophthalmol, 2006-09.

15. Hornig C, Barleon B, Ahmad S, Vuorela P, Ahmed A and Weich HA: Release and complex formation of soluble VEGFR-1 from endothelial cells and biological fluids. Lab Invest 80: 443-454, 2000.

16. de Araújo CE, Ribeiro MS, Favaro R, Zezell DM and Zorn TM: Ultrastructural and autoradiographical analysis show a faster skin repair in He-Ne laser-treated wounds. J Photochem Photobiol B 86: 87-96, 2007.

17. Shen Y, Fang C, Huang Y, Li Z, Xue Z and Li Y: Effects of low-power semiconductor laser irradiation on healing of tympanic membrane perforations of guinea pigs. Chin J Laser Med Surg 7: 386-392, 2008 (In Chinese). International (CC BY-NC-ND 4.0) License. 